\title{
Cambios en el desempeño profesional de la inspección educativa en España en los últimos cincuenta años
}

\begin{abstract}
Resumen
El objetivo principal de este estudio es analizar los cambios efectuados en la Inspección educativa española desde el impulso tecnocrático que se llevó a cabo en el periodo franquista, para así comprender mejor su ubicación en el sistema educativo vigente. En la actualidad, este es un órgano de la administración educativa presente en todas las comunidades autónomas del que no existen dudas manifiestas sobre su utilidad, lo que puede percibirse como signo de larga continuidad, al haberse convertido en una herramienta imprescindible para favorecer el aumento de la calidad educativa.
\end{abstract}

Palabras clave: Inspección Educativa. Sistema Educativo Español. Legislación. Funciones. Atribuciones.

\section{Erika González García}

Universidade de Granada -

Espanha

erikag@ugr.es

\author{
Antonio Salmerón Nieto \\ Universidade de Granada - \\ Espanha \\ asalmeron@ugr.es \\ Miguel Beas Miranda \\ Universidade de Granada - \\ Espanha \\ mbeas@ugr.es
}

\footnotetext{
Para citar este artigo:

GARCÍA, Erika González; SALMERÓn NIETO, Antonio; MIRANDA, Miguel Beas. Cambios en el desempeño profesional de la inspección educativa en España en los últimos cincuenta años. Revista Linhas. Florianópolis, v. 18, n. 36, p. 221-250, jan./abr. 2017. Revisado por Diana Sorgato.
} 


\section{Mudanças no desempenho profissional da inspeção educativa na Espanha nos últimos cinquenta anos}

\begin{abstract}
Resumo
O objetivo principal deste estudo é analisar as mudanças havidas na Inspeção educativa espanhola desde o impulso tecnocrático levado a cabo no período franquista, para assim compreender melhor sua situação no sistema educativo vigente. Trata-se, hoje, de um órgão da Administração Educacional presente em todas as comunidades autônomas, a respeito do qual não existem dúvidas manifestas quanto a sua utilidade, o que pode ser percebido como indicação de longa continuidade, ao ter-se convertido em uma ferramenta imprescindível para favorecer $\mathrm{o}$ aumento $\mathrm{da}$ qualidade educativa.
\end{abstract}

Palavras-chave: Inspeção Educativa. Sistema Educacional Espanhol. Legislação. Funções. Atribuições.

\section{Changes in the professional performance of the educational inspection in Spain during the last fifty years}

\begin{abstract}
The main objective of this study is to analyze the changes that happened in the Spanish educational Inspection since the technocratic impulse that was produced in the Francoist period, so that we may better understand its situation within the educational system presently in force. It is today an agency of the Educational Administration, with presence in every one of the autonomous communities, regarding which there are no manifest doubts as to its usefulness, what can be noticed as an indication of long continuity, as it converted into an essential tool to favor the raise in the quality of education.
\end{abstract}

Keywords: Educational Inspection. Spanish Educational System. Legislation. Functions. Assignments 
La Inspección de Educación cuenta, en su estructura contemporánea, con más de 160 años de existencia. A lo largo de ese tiempo, sus funciones y, con base en estas, las atribuciones y competencias, han ido evolucionando acordes a las necesidades del sistema educativo y, en ocasiones, siguiendo los intereses de los diferentes grupos políticos en el ejercicio del poder.

Resulta de interés abordar el estudio del desempeño profesional con objeto de analizar la trayectoria seguida para poder comprender mejor su ubicación en el sistema educativo actual, e incluso, el porqué de los cambios realizados en la asignación de desempeños profesionales. Cabe señalar que, en la actualidad, este es un órgano de la administración educativa presente en todas las comunidades autónomas de España del que no existen dudas manifiestas en cuanto a su utilidad.

El presente documento interviene las funciones asignadas a la Inspección educativa y aborda también las consecuencias que los cambios en las atribuciones y competencias y de ubicación en el organigrama de la administración producen en los componentes del cuerpo de Inspección, sobre todo la situación de conflicto vivida en la etapa final del franquismo.

\section{1 - Funciones de la Inspección profesional a lo largo de su Historia}

El nacimiento de la Inspección educativa mediante Real Decreto de 30 de marzo de 1849, conlleva el desarrollo legislativo correspondiente y en su seno el establecimiento del Reglamento para los Inspectores de Instrucción Primaria del Reino (REAL DECRETO DE 20 MAYO DE 1849), en que se señalan básicamente las funciones de vigilar el cumplimiento de las leyes educativas, informar a la superioridad, visitar las escuelas y asesorar a los maestros (SUREDA, 2006).

La relación anterior estará presente en los cometidos de la Inspección hasta llegar a la actualidad, aunque a estas funciones se sumarán la imposición de medidas disciplinarias (REAL DECRETO DE 25 DE AGOSTO DE 1885) la supervisión escolar (DECRETO 2915/1967) y la evaluación (DECRETO 664/1973).

La ejecución del conjunto de funciones implica la posibilidad de asignar a la Inspección toda una serie de atribuciones y competencias que la convertirán, a lo largo de 
más de un siglo, en los ojos, manos y pies de la administración educativa estatal en las diferentes provincias. De hecho, en el preámbulo del Real Decreto de fundación de la Inspección profesional se expresa: «Si en todos los ramos del servicio público es conveniente esta clase de funcionarios, en la instrucción primaria es indispensable. Sin ellos la administración nada ve, nada sabe, nada puede remediar» (MAILLO, 1989, p. 66). Esta situación de privilegio convirtió a este cuerpo en el auténtico órgano de la administración educativa a nivel provincial situándolo en una posición de poder que, de algún modo, excede la dimensión funcionarial e invade, en determinados momentos, la dimensión política.

La situación referida encuentra su límite en los años 60, en que la ocupación del poder por la tecnocracia del régimen franquista genera un nuevo organigrama administrativo que da lugar a la creación de las delegaciones provinciales de los diferentes ministerios y, por ende, del Ministerio de Educación y Ciencia. Serán órganos de administración dirigidos por el poder político a los que quedarán sometidos los miembros del cuerpo de Inspección, configurándose como un servicio específico de dichas delegaciones.

Esta nueva definición administrativa produce cambios en las atribuciones y competencias conferidas por las funciones de la Inspección. Las funciones siguen siendo las mismas, e incluso aumentan, pero las atribuciones y competencias cambian y hasta se puede decir que disminuyen en determinados desempeños. No obstante, se intensifican en otros.

Se entra en una etapa de redefinición del servicio de Inspección que se prolonga hasta 1995, momento en que se consigue definir y asentar un modelo que continúa vigente hasta la actualidad y que acaba con los conflictos que se iniciaron en los años 60, se mantuvieron en los 70, debido a los cambios orgánicos y funcionales, y se extendieron a los 80 y a la primera mitad de los 90, en virtud del cambio de modelo de Inspección, que pasa de la concepción de «Cuerpo» a la de «Función». 


\section{2 - Crisis de la inspección de Educación Primaria en la etapa del desarrollismo del régimen franquista}

La vida profesional del cuerpo de Inspección creado en 1849 se desarrolla durante más de un siglo en un proceso de vaivenes de alza y baja, sometidos al albur de los contextos políticos que, en conjunto, contribuyeron a prestigiar y potenciar poco a poco la posición administrativa de que gozaba.

La llegada de las delegaciones a partir de 1967, y el proceso seguido para su configuración, pondrán de manifiesto la percepción de inicio del sometimiento del cuerpo de Inspección de educación al poder político, confiriéndole a cambio mayor capacidad técnica. De hecho, desde 1957, aparecen medidas de acercamiento de la Inspección a la escuela con una nueva normativa de visitas y la creación de las inspecciones comarcales (ASOCIACIÓN DE INSPECTORES DE EDUCACIÓN DE ANDALUCÍA, (s.f)).

En la dirección del Ministerio de Educación de Manuel Lora Tamayo (1962 - 1968), se localizan bastantes indicios de un arrinconamiento progresivo de la Inspección y su sustitución por Técnicos de la Administración Civil (TAC), formados en la Escuela Nacional de Funcionarios (ASOCIACIÓN DE INSPECTORES DE EDUCACIÓN DE ANDALUCÍA, (s.f)).

No obstante lo expresado, se ha de señalar la aprobación de un nuevo Reglamento de Inspección en 1967 que sustituye el de 1932, gestado durante el Bienio Progresista de la II República, vigente durante gran parte de la Dictadura de Franco en virtud de su especial dimensión técnica. Entre las características que lo definen, cabe resaltar, según Leónidas Gonzalo Calavia, que «queda perfectamente configurada la misión de la Inspección, atribuyéndole las funciones de velar por la observancia de las disposiciones legales en toda clase de centros y asumiendo, además, las tareas de supervisión, dirección técnica, y orientación pedagógica de la enseñanza y de los servicios circumescolares (servicios complementarios)» (LÓPEZ, 2013, p. 506).

Es la primera vez que se utiliza el concepto de «supervisión» como función de la Inspección. Hasta este momento, entre las funciones, figuraban «vigilar el cumplimientos de la leyes» o «controlar el funcionamiento del sistema educativo». En esta etapa, se 
introduce este nuevo concepto tomado de la literatura pedagógica anglosajona que aporta una dimensión diferente, menos rígida y autoritaria.

A continuación, se aborda el tratamiento de procesos significativos desarrollados en el sistema educativo en los años sesenta y setenta, con incidencia en la redefinición orgánica y funcional de la Inspección educativa. Concretamente se interviene la puesta en marcha de las delegaciones provinciales, la creación de los institutos de ciencias de la educación, la elaboración de la Ley General de Educación (LGE) y su implementación.

\section{1 - Las delegaciones provinciales del Ministerio de Educación y Ciencia}

En 1967, se inicia un proceso de reorganización de la Administración Civil del Estado con el objetivo de reducir el gasto público en el marco del Plan de Estabilización económico y político (BEAS, 1999). Dentro de ese proceso de reorganización, tiene lugar el nacimiento de las delegaciones provinciales del Ministerio Educación y Ciencia (DECRETO 2538/1968) en las que se integrará el personal y los órganos del Ministerio de cada provincia y, por ende, el servicio de Inspección. Beas Miranda señala que «los Tecnócratas del tardofranquismo actuarán pretendiendo controlar todos los resortes del poder. La Inspección, por su parte, como cuerpo, no podía ensombrecer al poder político pero, tampoco estaban dispuestos sus miembros a desprenderse fácilmente del prestigio, del reconocimiento social y del poder acumulados desde hacía más de un siglo» (BEAS, 1999, p. 356). El conflicto estaba servido.

La sustitución del ministro Lora Tamayo se lleva a cabo en la persona de José Luís Villar Palasí, mediante decreto de 16 de abril de 1968, en una época en la que se realizan cambios importantes que tendrán incidencia en la Inspección. Entre los cuales, la creación de las delegaciones provinciales de Educación y Ciencia, mediante Decreto de 27 de noviembre de 1967, en cuyo artículo 7.4 se expresa que «Sin perjuicio de las atribuciones de las autoridades académicas, se refunden los servicios administrativos dependientes del departamento en cada provincia en una Delegación Provincial de Educación y Ciencia...».

El Decreto de 25 de septiembre de 1968 regula las delegaciones provinciales. En estas habían de integrarse todas las jefaturas y delegaciones de servicios existentes en las provincias, entre las cuales, las de Inspección, lo que supone un cambio de estatus, ya que 
dejarán de depender directamente de la correspondiente dirección general del ministerio para hacerlo de la delegación. Es algo, que supone un descabalamiento en la importancia orgánica que no será fácilmente aceptado (BEAS, 1993).

En este proceso, llama la atención el hecho de que el nuevo equipo ministerial no diera audiencia a las inspecciones técnicas en la elaboración de normativa que tanto le afectaba; lo que motivó la dimisión del Inspector General de Enseñanza Primaria (LÓPEZ, 2013).

La nueva estructura organizativa enmarca a la Inspección técnica subordinada orgánicamente, al Ministro, al Subsecretario o al Director General de Ordenación Educativa y funcionalmente al Delegado Provincial, sin quedar claro, en principio, cómo se conjugaban esa relación de dependencia a la hora de ejercer sus funciones diariamente (BEAS, 1999). Pero, en definitiva, los delegados, representantes del Ministro en la provincia, ejercerán su autoridad en todo el ámbito de la delegación del Ministerio y, por tanto, sobre la Inspección técnica provincial de Educación General Básica (EGB).

Por otro lado, cabe señalar que la llegada de las delegaciones provinciales supone la creación de toda una serie de departamentos especializados ocupados por técnicos de la administración que vienen a ocupar atribuciones y competencias desempeñadas, hasta el momento, por la Inspección técnica de educación. Esto, evidentemente, contribuye a incrementar el malestar en el cuerpo por la pérdida de influencia y de prestigio que implícitamente ello supone. La eliminación de parcelas de intervención es entendida como una pérdida de confianza en la capacidad de gestión.

No obstante, se ha de entender que se entra en una fase expansiva del sistema educativo y de modernización de la administración del Estado que pretende la tecnificación de la gestión.

Ante la nueva etapa, se podría haber elegido entre dos fórmulas de abordaje: confiar al servicio de Inspección, altamente tecnificado, la gestión del sistema educativo a nivel provincial; o, dejarle la parcela específica referida a sus funciones clásicas, adaptadas a las necesidades del sistema educativo del momento. La primera podría haber sido una buena opción, puesto que contaba con los técnicos que mejor conocían el sistema, pero con el peligro de incrementar desmesuradamente su poder y su área de influencia, lo que, 
a lo largo del tiempo, podría ser contraproducente para el ejercicio del poder político. La opción adoptada permitía el sometimiento directo al poder de los delegados provinciales y la creación de departamentos específicos para la gestión de los diferentes ámbitos del sistema.

Pasado el tiempo, se puede afirmar que la decisión fue acertada, puesto que su esquema de funcionamiento se ha prolongado llegando a la actualidad en un proceso de desarrollo de las delegaciones provinciales, que ahora pertenecen a la Consejería de Educación de cada autonomía, en paralelo con el proceso expansivo que el sistema educativo ha tenido en los últimos cincuenta años. La Inspección de Educación sigue existiendo como cuerpo y servicio educativo perfectamente encuadrado en la estructura del sistema actual, en continuo proceso de adaptación de sus funciones, lo que produce, en muchos momentos, la percepción desde dentro de una tendencia a la relativización de dichas funciones. Sin embargo, no se ha de perder de vista que es un instrumento al servicio de la administración educativa, seguidora de determinadas directrices políticas y burocráticas a las que se debe adaptar. Pero lo realmente significativo, es que, en la configuración del actual organigrama de gestión, nadie duda de la necesidad de la Inspección para el adecuado funcionamiento del sistema y, más importante aún, para asegurar el incremento de la calidad de la educación de que tanto necesitan España, y Andalucía, en particular.

\section{2 - Los Institutos de Ciencias de la Educación}

La labor de la Inspección contaba con la función de asesorar al profesorado desde la misma creación del cuerpo. Dentro de esta función, se fue configurando la competencia para llevar a cabo la formación permanente en colaboración con las Escuelas Normales. Esta situación permanecerá hasta el nacimiento de los Institutos de Ciencias de la Educación (ICE), que tienen la misión de efectuar la formación pedagógica y el perfeccionamiento del profesorado, investigar en el sector de las ciencias de la educación y prestar asesoramiento técnico en los problemas educativos (LÓPEZ, 2013). A partir de aquí, la Inspección continúa colaborando con los ICE, pero sin el protagonismo 
de la etapa anterior. Ya ha nacido un nuevo órgano de la administración con la misión específica de intervenir estos temas.

\section{3 - La Ley General de Educación}

El nuevo equipo al frente del Ministerio de Educación y Ciencia se planteó abordar una reforma integral del sistema educativo como fórmula para dar respuesta a las necesidades que la sociedad española demandaba, fruto de su incorporación a las sociedades modernas de capitalismo avanzado. La respuesta a la situación existente se va a articular mediante la elaboración de una nueva ley de educación que será conocida como Ley General de Educación (LGE), de 1970 (LEY 14/1970).

Las labores para alumbrar la nueva ley se van a iniciar preparando a la opinión pública para aceptar los cambios que serán realizados. Esto se llevó a cabo mediante la presentación del «libro blanco», un documento abierto a todas las críticas y sugerencias, en que se realizaba un análisis de la situación existente en el campo de la educación y se diseñaban las nuevas líneas del sistema (LÓPEZ, 2013).

El documento citado, «La educación en España: bases para una política educativa» (MINISTERIO DE EDUCACIÓN Y CIENCIA, 1969), dedica a la Inspección el punto 135 y, sobre esta, manifiesta que «Las inspecciones de Educación General Básica y de Bachillerato se conciben como organismos técnicos que han de asegurar una eficaz programación y administración educativa. Asumirán la responsabilidad del control del rendimiento educativo y la dirección y orientación de la enseñanza. A ese efecto, será incrementado su personal y se les desligará de determinadas actividades administrativas que van en menoscabo de las funciones genuinamente técnicas que les competen».

La redacción permite apreciar una alta consideración sobre el papel del cuerpo de Inspección y, con base en esto, se le asignan funciones genéricamente importantes, pero al mismo tiempo, se menciona el abandono de otras menos técnicas pero asignadas desde largo tiempo y que probablemente han conferido prestigio y poder. Una definición en esta línea seguro que generará tensiones entre el Ministerio y los miembros del cuerpo, recelosos ante la percepción de un proceso de marginación. 
En otro documento encargado por el Ministerio a una comisión internacional de expertos (INFORME FINAL DEL COMITÉ DE COOPERACIÓN INTERNACIONAL PARA LA REFORMA DE LA EDUCACIÓN EN ESPAÑA, 1968, pp. 87-89), se apunta a un cuestionamiento de las funciones de la Inspección, ya que, al mencionar la evaluación del profesorado, se expresa que «Los inspectores no deben inspeccionar; fundamentalmente serán consejeros y consultores expertos que ayuden al maestro a resolver sus dificultades, a llenar sus lagunas y a actualizar sus potencialidades como tales maestros».

Estos cuestionamientos sobre las funciones de la Inspección serán fuente de tensiones y conflictos, que se agudizarán, «ante la evidente prevención que iban a mostrar los rectores de la reforma hacia los funcionarios que habían servido hasta ese momento en los cuerpos de Inspección» (INFORME FINAL DEL COMITÉ DE COOPERACIÓN INTERNACIONAL PARA LA REFORMA DE LA EDUCACIÓN EN ESPAÑA, 1968, p. 515).

No cabe duda que se está en un proceso de cambio general en el sistema educativo y, por tanto, todas sus estructuras se verán afectadas, provocando en ellas situaciones de defensa cuando se estima necesario y más en el servicio de Inspección que ya venía de una década con percepción de marginación en el desempeño profesional.

La posición del Ministerio quedará claramente manifiesta en el proyecto de ley presentado a las Cortes el 24 de octubre de 1969, en que se establece la constitución de un servicio de Inspección unificado (INFORME FINAL DEL COMITÉ DE COOPERACIÓN INTERNACIONAL PARA LA REFORMA DE LA EDUCACIÓN EN ESPAÑA, 1968, p. 515), acabando con la separación entre la Inspección de enseñanza primaria y de enseñanza media. Para que eso se aplique, habrá que esperar la Ley Orgánica de Participación, Evaluación y Gobierno de los centros educativos (LOPEGCE), en 1995. La cual generará tensión entre el Ministerio y la Inspección de enseñanza media, que se opondrá a la medida. La situación final contempla la creación de un Cuerpo Especial de Inspectores Técnicos de Educación, algo que no llegó a aplicarse en aquella época. En principio, porque los responsables del Ministerio no tenían interés en crear un cuerpo que en el fondo no habían deseado y habría supuesto, como se acaba de mencionar, el descontento de la Inspección de enseñanza media, que consideraba que se le habían usurpado los cuatro primeros cursos de Bachillerato para incorporarlos a la Educación 
General Básica (INFORME FINAL DEL COMITÉ DE COOPERACIÓN INTERNACIONAL PARA LA REFORMA DE LA EDUCACIÓN EN ESPAÑA, 1968).

\subsection{La Inspección en la implantación y desarrollo de la Educación General Básica}

«A pesar de la marginación de los inspectores en la elaboración de las normas legales, el Ministerio va a contar con ellos a la hora de hacerlas realidad» (INFORME FINAL DEL COMITÉ DE COOPERACIÓN INTERNACIONAL PARA LA REFORMA DE LA EDUCACIÓN EN ESPAÑA, 1968, p. 526).

La tarea asignada será extraordinaria, ocupada en distintos aspectos que se intervienen a continuación (INFORME FINAL DEL COMITÉ DE COOPERACIÓN INTERNACIONAL PARA LA REFORMA DE LA EDUCACIÓN EN ESPAÑA, 1968, pp. 526-527):

En el aspecto pedagógico se hace una amplia campaña de difusión y orientación de las innovaciones pedagógicas presentes en la nueva ley.

La formación del profesorado requiere, sobre todo, de la organización y participación de la Inspección en cursos para la implantación de la segunda etapa de la EGB.

Se le encomienda la autorización y seguimiento de las experimentaciones pedagógicas, con la creación de centros piloto y centros experimentales y, también, la experimentación de la aplicación del $5 .^{\circ}$ curso de EGB.

Colabora en la gestión del déficit de aulas para escolarizar en los colegios a los jóvenes de 10 a 14 años que, en la etapa anterior, se repartían entre la escuela y el instituto. La Inspección no tenía responsabilidad ni autoridad sobre ese tema, y menos desde la creación de las delegaciones, pero la especial situación en contacto con la realidad le convierte en parachoques de todas las demandas, protestas y reclamaciones.

Con el fin de ajustar la actuación de la Inspección a la etapa educativa que se empieza a vivir en España, se lleva a cabo una nueva regulación de las funciones y atribuciones que le aproximan a una dimensión más técnica (DECRETO 664/1973). No obstante, introducidas esas modificaciones (DECRETO 664/1973), el Reglamento de 1967 
sobrevive al fin de la Dictadura y a la llegada de Democracia. Irá desapareciendo con la configuración de las autonomías y la correspondiente asunción de competencias educativas. En particular, en Andalucía estará vigente hasta la regulación contenida en el Decreto de 1987 (DECRETO 65/ 1987).

Lo analizado permite una percepción bastante amplia de los principales aspectos en que se localizan cambios en la asignación de atribuciones y competencias en la etapa objeto de estudio. A continuación, se presenta de modo focalizado el desempeño profesional del equipo técnico de Inspección de Granada entre 1970 y 1977 con el objetivo de conocer el discurrir concreto de un servicio provincial, apreciando sus posibles similitudes con lo que hasta el momento se ha presentado de modo genérico.

\section{3 - Desempeño profesional del Equipo Técnico de Inspección de Granada entre 1970 y 1977}

El apartado que se presenta se origina de la tesis doctoral inédita realizada en 1993 por el profesor de Teoría e Historia de la Educación del Departamento de Pedagogía de la Universidad de Granada, D. Miguel Beas Miranda intitulada «Implantación y desarrollo de la EGB en Granada. Estudio de la creación y funciones de la Delegación Provincial de Educación (1970-1977)`». En esta, se dedica el capítulo IV a la Inspección Técnica Provincial de Educación de Granada, acometiendo un estudio que abarca aspectos como la Inspección Técnica Provincial en la Ley General de Educación de 1970, problemas entre Inspección y Delegación, trabajo informativo, formativo y valoración del profesorado por parte de la Inspección; dificultades económicas, burocráticas y administrativas para construir, crear o suprimir aulas y dotarlas con personal docente, ponencias e Inspección y recursos económicos.

El tratamiento del conjunto de aspectos presentará la visión global del desempeño profesional de la Inspección y las condiciones y circunstancias en que se desarrolla en el periodo objeto de análisis, 1970 - 1977.

Su inclusión pretende el acercamiento a la situación vivida en estos años con el objetivo de permitir una más adecuada percepción de los hechos relatados como genéricos, mediante la presentación de una ubicación concreta. En un momento en que 
se encuentra en fase de creación la delegación provincial y la correspondiente integración en esta del servicio de Inspección. A la vez, en paralelo, se lleva a cabo la implantación progresiva de la Ley General de Educación. Ambos hechos permiten señalar la importancia de la etapa en proceso de estudio.

Se trata de un periodo en muchos casos señalado como el más importante para la Inspección en los últimos cincuenta años, ya que supone la entrada en una fase de adaptación permanente de la labor inspectora a las demandas del sistema educativo, quedando insertada como una herramienta de la administración al servicio de la mejora de la calidad educativa. Son cambios que se suceden a través del tiempo, percibidos por diversos componentes como una acción de descabalamiento del poder adquirido e incluso con la intención de la eliminación del cuerpo, pero el tiempo transcurrido permite apreciar que se van llevando a cabo cambios en las funciones y atribuciones, de modo periódico, que buscan dar respuesta a las demandas que el sistema impone a la Inspección. De algún modo, se puede ver como una herramienta que se ocupa de atender, mediante la administración y supervisión, los aspectos de expansión del sistema educativo, que al convertirse en elementos maduros del sistema, pasan a ser atendidos por servicios específicos creados ad hoc, dejando de ser atribución correspondiente al ejercicio de las funciones de la Inspección (algo que ocurre a lo largo del tiempo con la creación de centros, la dotación de unidades, de materiales didácticos, recursos humanos, jefatura provincial de educación, cupos de profesorado, escolarización, formación del profesorado, asesoramiento, e incluso últimamente con evaluación). Se ha de entender que si no se efectuara la creación de los mencionados servicios específicos, el de Inspección se convertiría en un macroservicio, ocupado intensamente en labores administrativas y escasamente en labores técnicas de supervisión del sistema.

El modelo aplicado a lo largo de los últimos cincuenta años, permite apreciar hoy en día una Inspección integrada en las delegaciones provinciales, con unas dimensiones en constante crecimiento y en equilibrio con el resto de servicios. De tal modo, que se ha evitado una macrocefalia que podría haber dificultado, por el ejercicio monopolístico, la acción del poder político. Hay que tener presente, que la Inspección siempre ha contado con la mejor preparación técnica para intervenir en el sistema educativo y, por ello, ha reivindicado su participación en la gestión de los cambios fruto de la expansión pero, en 
muchas ocasiones, en los últimos tiempos, se le ha negado dicha participación, por el peligro que suponía poner bajo su responsabilidad una porción excesiva del andamiaje educativo.

\subsection{Relaciones entre la Delegación y la Inspección}

Este tema se aborda mediante el epígrafe PROBLEMA INSPECCIÓN-DELEGACIÓN. Este sitúa de lleno y sin dilaciones el estado de las relaciones. Los aspectos legales del problema se ubican, en principio, en la escasa claridad de la normativa a la hora de definir competencias y jerarquías lo que da lugar a que, en determinados momentos, no se sepa a quién se está subordinado a la hora de cumplir un cometido (BEAS, 1993).

Esta situación, según manifestaciones de miembros del servicio (BEAS, 1993), produjo en Granada cierto conflicto, al menos a nivel dialéctico, entre la Inspección y la Delegación. A ello, contribuyó también el hecho de que la ubicación física de ambos órganos no coincidiese en el mismo edificio hasta 1978. La separación física era percibida como negativa, ya que dificultaba la inmediatez de la comunicación y el sentido de pertenencia a un mismo ente (BEAS, 1993).

Por otro lado, cabe señalar que los miembros del cuerpo sienten con mayor crudeza el descenso de su influencia y de poder cuando sus funciones pasan a ser desempeñadas por otro personal, como en el momento en que la máxima autoridad administrativa provincial en educación, pasa a ser la jefatura de la Delegación y no la jefatura de Inspección (BEAS, 1993).

La nueva situación requiere de un reajuste que suponía adaptar el trabajo autónomo a nivel provincial al nuevo orden, lo que produjo algunos casos de conflicto personal, aunque, según las distintas partes, de escasa importancia.

Otra aspecto, fue la actitud crítica que tuvo la Inspección ante la forma de implantación de la Ley General de Educación en Granada, ya que su preparación técnica le hacía disentir en muchos momentos de las decisiones tomadas por la autoridad competente (BEAS, 1993). Decisiones que la Inspección no compartía, pero que respetaba. 


\subsection{1 - Quejas y reproches de la Inspección a la Delegación}

En otro orden de asuntos, las relaciones entre la Inspección y la Delegación presentan una serie de quejas y reproches de la primera hacia la segunda que se concretan con respecto al profesorado (BEAS, 1993) en el poco caso que se le hace a la solicitud de datos sobre diferentes cuestiones y en la no intervención en la selección del profesorado de las Escuelas Hogar. Por otro lado, se presentan quejas y reproches debidos a las construcciones escolares, a la escolarización (BEAS, 1993), a la incorrecta interpretación normativa y a la difusión del contenido de algunos informes.

El conjunto de las cuestiones presentadas permite concluir la existencia de tensiones en las relaciones, que se deben, según la propia Inspección Técnica Provincial, a la pérdida de poder y de capacidad de influencia en las decisiones de la Delegación Provincial de Educación (BEAS, 1993).

No obstante lo anterior, cabe señalar la participación y colaboración de la Inspección con la Delegación, tanto por voluntad propia como por solicitud, en casos (BEAS, 1993) de formación de comisiones, planificación escolar y situación educativa de todas y cada una de las localidades de la provincia, así como puntualmente en el estudio de las mejoras que podrían conseguirse con inversiones de reducida cuantía.

En la investigación se llega a deducir, tanto por el testimonio de la Inspección como por el de la Delegación, que la colaboración, cuando la hubo, fue muy eficaz.

\section{2 - Protestas de la Inspección motivadas por recortes en sus atribuciones}

El prestigio de la Inspección Técnica se fundamentaba, entre otros motivos, en su dependencia orgánica del Subsecretario del MEC y por haber ejercido, durante mucho tiempo, una serie de funciones desglosadas en atribuciones que le posibilitaban la consecución de poder y prestigio indiscutidos en el contexto educativo. La llegada de la etapa que es objeto de análisis, como se viene manifestando, hace saltar las alarmas y, como se percibe, la reacción de protesta para la conservación del estatus no se hace esperar, expresándose en cada momento que se considera la afección por recorte en las atribuciones. Son situaciones que se presentan con la (BEAS, 1993): 
- $\quad$ Supresión de la presidencia por la Inspección de los tribunales de oposición de Magisterio.

Pérdida de la posibilidad de comunicación directa con los órganos de la Administración central, que pasa a hacerse a través del Delegado Provincial

Exclusión de la Inspección Técnica de Educación del organigrama de la Administración Central e inclusión en el organigrama de la Delegación Provincial.

El problema de fondo, es posible que radique en que después de la aprobación de la LGE no se definen claramente las funciones y atribuciones de la Inspección, no se arbitren medidas para que puedan cumplirlas y no se aclare su situación en el organigrama administrativo, así como sus relaciones con los representantes políticos.

A pesar de ello, se ha de entender que se somete a la Inspección a un descabalamiento jerárquico que esta difícilmente aceptaría por muchas reglamentaciones normativas que se hiciesen, ya que la ambigüedad siempre permitiría el resquicio para la reivindicación de su preeminencia técnica y funcionarial, muy superior a la de cualquier representante político.

\section{3 - Desempeño de otras atribuciones}

Esta sección, trata de las atribuciones que en el periodo objeto de estudio tuvo la Inspección y que, en la actualidad, ha dejado de ejercer o las interviene con otros procedimientos. Se refiere a intervenciones en la conservación de los centros, información y formación del profesorado y distinciones y sanciones de la Inspección respecto al profesorado.

\subsection{1 - Actuaciones en la conservación de los centros}

La normativa sobre construcciones escolares establecida a partir de la Ley de Construcciones escolares de 1953, establecía la obligatoriedad de los ayuntamientos de llevar a cabo el mantenimiento y la conservación de los edificios escolares y de las casas de los maestros. Sin embargo, la escasez de recursos de los ayuntamientos granadinos hace que la mayor parte de ellos releguen sus obligaciones. 
Por consecuencia, para solucionar ese grave problema, la Inspección presenta denuncia ante la Delegación, para que esta recuerde a los ayuntamientos sus obligaciones y presione políticamente a las jerarquías de orden superior. En otros momentos, busca la colaboración de las familias, interesadas directas en que sus hijos estén bien atendidos, para paliar las negligencias de la administración educativa y de los ayuntamientos.

Evidentemente, la conservación de los edificios escolares no es una cuestión técnica que compita al ámbito de intervención de la Inspección, pero no cabe duda de que su estado influye en la calidad de la educación que recibe el alumnado y, en ese sentido, se han de entender las acciones mencionadas.

\subsection{2 - Actuaciones para construir, habilitar o suprimir aulas y dotarlas con personal docente}

«Una vez aprobada la LGE, la Inspección, lo mismo que la Delegación Provincial, examinó el tema de la escasez de aulas y el estado de los edificios escolares» (BEAS, 1993).

Obsérvese que la redacción del texto manifiesta la realización de acciones paralelas, por la Inspección y la Delegación, sobre el estudio de la situación de edificios y aulas. No hay expresión de una acción coordinada, lo que puede estimarse como señal del estado de las relaciones entre ambos organismos que posiblemente actúan de modo independiente, cuando lo normal, según el funcionamiento actual, es que la primera sirva de fuente de información a la segunda al estar plenamente integrada en su organigrama.

Desde la Inspección se pretendió colaborar eficazmente incluso denunciando frecuentes anomalías que se cometían en las construcciones escolares, a las que se daba escasa respuesta. Por ello, dolía el poco caso que se hacía a sus informes y la marginación a la que era sometida, según quejas de los propios miembros (BEAS, 1993). 


\subsection{3 - Actuaciones de información al profesorado}

Las actuaciones de información al profesorado se realizarán mediante tres vías: visitas y reuniones, documento de publicación periódica denominado Boletín de Educación y circulares.

\subsubsection{1 - Boletín de Educación}

El Boletín de Educación era redactado y editado por el propio servicio de Inspección e incluso sufragado con sus propios fondos, aunque a partir de 1974 se solicita que lo haga la Delegación. Era un trabajo suplementario que se llevaba a cabo para apoyar a los centros, y el carácter anónimo de gran parte de los artículos escritos resaltaba el carácter colectivo de la publicación (BEAS, 1993).

Con respecto al contenido, se aprecian dos etapas: una de difusión de normativa y orientaciones de tipo pedagógico y administrativo; y otra, de divulgación de experiencias pedagógicas.

Se considera que la publicación del Boletín supuso un gran esfuerzo en el ejercicio de la función de asesoramiento al profesorado.

\subsubsection{2 - Circulares}

La redacción de circulares se llevó a cabo, en ocasiones, para informar a los centros sobre algunos aspectos o solicitar algún tipo de información. Normalmente no plantearon ningún problema a la comunidad escolar, aunque hubo una cuestión referente al horario de trabajo, que produjo gran malestar entre el profesorado (BEAS, 1993).

\subsection{4 - Actuaciones de formación del profesorado}

Estas actuaciones están encuadradas en la colaboración con el Instituto de Ciencias de la Educación y la intervención en los Centros de Colaboración Pedagógica, y marcan el inicio de la etapa de alejamiento de la Inspección con respecto a las actividades de formación permanente del profesorado. 


\section{3·3.4.1 - Relaciones con el Instituto de Ciencias de la Educación}

Las actuaciones de formación del profesorado se encuentran con el entramado teórico de la desviación normativa que establece confluencia de funciones entre la Inspección y los ICE, aunque esto no llega a producir un trastorno, ya que ambos organismos se esfuerzan por establecer una colaboración fructífera (BEAS, 1993).

La implantación de la LGE dará pie a una etapa de gran colaboración, convirtiéndose en el tema clave de la relación. La Inspección, por su parte, constituye un equipo de trabajo con la participación de todos los miembros del servicio provincial, con el objetivo de colaborar para el perfeccionamiento del profesorado (BEAS, 1993).

\subsubsection{2 - Los Centros de Colaboración Pedagógica}

Son un medio promovido por la administración y puesto a disposición de la Inspección para el perfeccionamiento del profesorado. Cuentan con cierta tradición en la enseñanza primaria. Son reflejos de etapas anteriores cuyas raíces las encontramos en Giner de los Ríos. Sus funciones fueron reactualizadas mediante Orden Ministerial de 22 de octubre de 1957 , en la cual se señala que son constituidos por «grupos de maestros nacionales de la misma o distintas localidades, organizados y dirigidos, dentro de cada provincia por la Inspección respectiva para reunirse periódicamente, intercambiando doctrinas y experiencias... » (BEAS, 1993).

La percepción de la Inspección sobre el éxito del funcionamiento de los Centros de Colaboración Pedagógica indica que esta se encontraba mediatizada, puesto que, la demanda del profesorado chocaba con la escasez de recursos económicos. De ahí que el protagonismo que tenía la Inspección Técnica en la formación continua se va perdiendo a medida que los Centros tienden a desaparecer debido a la carencia de ayuda oficial, mientras que los ICE pasan a controlar por completo todas estas actividades. 


\subsection{5 - Actuaciones de distinción y sanción al profesorado}

Estas actuaciones son la cara y la cruz de una misma moneda. Actualmente, siguen siendo competencia de la Inspección la propuesta de apertura e instrucción de expedientes disciplinarios.

\subsubsection{1 - Distinciones}

Las distinciones de la Inspección al profesorado se realizaban mediante «voto de gracia», es decir, un reconocimiento o premio a la calidad y buen hacer profesional que se añadía a su expediente laboral. Normalmente se realizaban por acuerdo del Consejo de Inspección, mediante propuesta de la Inspección de referencia o instancia de la persona interesada. Casi siempre conllevaban un punto valedero para el concurso de traslados. En ocasiones la concesión tenía carácter colectivo, a todo el profesorado de un centro.

Las razones alegadas podían ser la labor desarrollada, los méritos profesionales, tener establecida la Obra Misional de la Santa Infancia, tener implantados en la escuela los medios audiovisuales, haber sido Jefe Local de Juventudes, etc.

Era un premio moral que se concedía a unos profesionales mal pagados que dedicaban horas extras sin remunerar, en nombre de lo que se entendía como «vocación docente».

No existía baremo para establecer los requisitos para su concesión. Quedaba a criterio de la Inspección de referencia y del Consejo de Inspección y, por tanto, cargado de subjetividad. Se planteó su elaboración pero no se llegó a concretar (BEAS, 1993).

La condecoración más importante para el profesorado de EGB en la que intervenía, de manera decisiva, la Inspección era la Cruz de Alfonso X, El Sabio, también denominada Cruz al Mérito Docente. 


\subsubsection{2 - Sanciones}

La función punitiva de la Inspección casi no se ejerce en el periodo objeto de estudio. Se realizan apercibimientos por diferentes motivos. Es una función reglamentada que se verá sometida a polémica, puesto que llega un momento, en 1975, en que el Delegado envía un escrito al jefe de Inspección en que pide que la imposición de apercibimientos con pérdida de haberes sea propuesta ante su autoridad para la resolución oportuna. Estimaba que el servicio de Inspección se debía limitar a proponer sanciones de descuento de haberes y que era su competencia confirmar o denegar la propuesta.

El planteamiento que se realiza es percibido por la Inspección como un nuevo recorte de poder, lo que resulta en protestas ante las autoridades provinciales y centrales del Ministerio, al entender que se transgredía la legalidad vigente por impedir que se ejerciesen todas las funciones que tenía encomendadas (BEAS, 1993).

\section{4 - Ponencias}

La utilización del término «Ponencia» se ha de entender como referencia a cada una de las áreas especializadas del sistema educativo que era atendida por un miembro del servicio de Inspección que actuaba como experto encargado de dicha área.

El número de ponencias era facultativo a cada Inspección Técnica Provincial, aunque estas debían ser sometidas por el Consejo de Inspección a la aprobación de la Dirección General de Enseñanza Primaria.

Se adoptará una gran flexibilidad organizativa al estructurar las ponencias en función de las necesidades educativas provinciales, con lo que se acometerán cambios en diferentes momentos.

El conjunto del funcionamiento de las ponencias, según se manifiesta en el estudio, se mueve entre la desorganización y la ineficacia, lo que repercute directamente en el prestigio del trabajo realizado, así como en su autoestima, dando lugar al desencanto y a la falta de rendimiento laboral (BEAS, 1993). 


\section{5 - Inspección y recursos económicos}

El desempeño de la labor inspectora en el periodo analizado se desarrolla mediatizado por las circunstancias económicas del país, que a partir de 1973 se ve inmerso en la crisis económica mundial derivada del aumento de los precios del petróleo, resultante de la actuación de la Organización de Países Exportadores (OPEP), cuya repercusión negativa en los presupuestos destinados a la educación no cambiará hasta los acuerdos de los Pactos de la Moncloa de 1977.

En el servicio de Inspección, la situación se manifiesta principalmente en las reclamaciones para conseguir el cobro de dietas y gastos de kilometraje. Son numerosos las quejas y los escritos realizados. En respuesta, la Administración establecerá un plan de control de las visitas de Inspección que perjudicará la autonomía y flexibilidad en el ejercicio de sus funciones. Con ello encontramos un nuevo elemento de fricción y restricción en el desempeño de la actividad profesional (BEAS, 1993).

\section{6 - Conclusiones sobre el servicio de Inspección en Granada}

1. Se constata que el vacío legal denunciado por la Inspección para el desarrollo de sus funciones no corresponde a la normativa vigente, ya que estas funciones se encuentran bien articuladas.

2. El poder del cuerpo cambia de forma notoria a raíz de la creación de las delegaciones provinciales de Educación. Ha de adecuarse para ejercer un nuevo papel en el sistema educativo.

3. La Inspección no se resigna a aceptar de forma pasiva lo que los representantes de la Administración Central o Provincial les ordenan.

4. La Inspección denuncia la dejación de los ayuntamientos en la conservación y mantenimiento de los centros.

5. Existió colaboración con el ICE que fue de mayor a menor intensidad en el periodo de estudio y que dependió de la actitud individual. 
6. El protagonismo en la formación del profesorado mediante los Centros de Colaboración Pedagógica se fue diluyendo hasta su desaparición debida a la pérdida de ayuda oficial.

7. La Inspección, según su propia apreciación, desempeñó un importante papel en la implementación de la LGE debido a su responsabilidad profesional, trabajo en equipo, capacidad crítica, autopreparación técnica, prestigio ante la comunidad educativa, relaciones cordiales con el profesorado y su alto grado de autoestima.

En todo el proceso que se acaba de presentar, se percibe cierto grado de corporativismo frente a diversos colectivos u organismos que, según la propia Inspección, mermaban su autoridad. Caber resaltar que se pasa de una etapa de máxima potencialidad, de máxima autoridad en la gestión del sistema educativo a nivel provincial; a otra, de descabalamiento motivada por cambios en la estructura organizativa de la Administración, que le llevan a ocupar una posición diferente y a ajustes periódicos con el propósito de adaptarla a las necesidades del sistema con base en las directrices establecidas desde el poder político.

Se puede apreciar, de forma clara y manifiesta, la conexión y coincidencia existente entre el desempeño profesional del servicio provincial de Inspección de Granada y la situación referida al conjunto de España, con la oportunidad de apreciar muchos más matices de expresión de desajuste e incomodidad en el enfoque realizado, puesto que aproxima de forma más objetiva, a la situación vivida. Aunque por un lado limita lógicamente la extracción de conclusiones generales, por otro lado, permite fundamentarlas.

\section{4 - Funciones de la inspección educativa en la España Democrática}

El proceso de reubicación de la Inspección técnica y sus funciones en el organigrama del sistema educativo contará con nuevos episodios en la España Democrática.

En principio, se ha de citar que será el artículo 27.8 de la Constitución de 1978 el que establece que «Los poderes públicos inspeccionarán y homologarán el sistema educativo para garantizar el cumplimiento de las leyes». Esta redacción, sin duda, pone de 
manifiesto que la acción inspectora está contemplada en la norma de más alto rango del ordenamiento jurídico de España. Ahora queda por ver cómo esta se articula en la nueva etapa política.

Es interesante señalar que la llegada del Régimen Democrático no supone el abordaje de cambios significativos en las funciones, ni por supuesto rupturas, ya que el Reglamento de Inspección de 1967 continuará vigente a lo largo de más de veinte años, estando ya bien asentada la Democracia. Dicho reglamento dejó de utilizarse en Andalucía tras la promulgación del Decreto de 1987 (DECRETO 65/ 1987) y, en las comunidades dependientes del Ministerio de Educación, en 1989, tras la publicación de un nuevo reglamento (REAL DECRETO 15/1989). No obstante, lentamente la Inspección Educativa seguirá perdiendo atribuciones y competencias que tradicionalmente se encontraban bajo su responsabilidad.

Por otro lado, cabe resaltar, que las modificaciones sustanciales planteadas por la Ley General de Educación de 1970 para la Inspección no se plasmarán completamente hasta 25 años después con la LOPEGCE en 1995 (LEY ORGÁNICA 9/1995), cuando se contempla la creación de un cuerpo unificado para Educación Primaria y Secundaria de Inspección Educativa (CUERPO DE INSPECTORES AL SERVICIO DE LA ADMINISTRACIÓN EDUCATIVA, 1984).

La política educativa desarrollada en los años de la Transición, con los gobiernos de UCD, continuó con el proceso de recorte de competencias en clave de trasvase de poder en el contexto de transferencia de competencias en la configuración de España en comunidades autónomas. La consolidación y el desarrollo de las delegaciones provinciales contribuye a que atribuciones en materia de planificación, personal, y otras, vayan siendo asumidas por servicios surgidos en su organigrama.

En otra dimensión, se lleva acabo el primer intento de planificar la actividad inspectora en todo el Estado, se aborda la homologación de la actuación y se regula la visita de Inspección (ASOCIACIÓN DE INSPECTORES DE EDUCACIÓN DE ANDALUCíA. (s.f)).

Son medidas destinadas a pronunciar la dimensión técnica de la actuación inspectora, pero que, a la vez, son percibidas como fórmulas de control que redundan en 
una limitación de la autonomía de intervención individualizada. Por lo que, dichas medidas son recibidas, hasta cierto punto, como parte del cerco de control y limitación que se viene estableciendo al poder de la Inspección.

En octubre de 1982, el PSOE llega al poder del gobierno del Estado. Se inicia una etapa de transformación del modelo de Inspección educativa proveniente del régimen franquista. Se pretende acabar con el Cuerpo de Inspección Educativa e introducir un nuevo concepto, el de Función Inspectora, concebida como un desempeño temporal (ASOCIACIÓN DE INSPECTORES DE EDUCACIÓN DE ANDALUCÍA. (s.f).). Se encuentra oposición de gran parte de los integrantes del cuerpo que lo perciben como el golpe definitivo para su exterminio, tras largos años de lenta, pero ineludible marginación y postergación. La transformación del modelo se verá plasmada en la LMRFP de 1984 (LEY 30/1984).

Cabe entender, aquí, que el Cuerpo de Inspección es percibido por el nuevo partido en el poder como un organismo anclado en el régimen anterior, una rémora para acometer las grandes transformaciones educativas que el nuevo gobierno tiene programadas.

De hecho, se cambia la forma de acceso, que no será por oposición, sino por selección mediante concurso de méritos, lo que facilitará adaptar los baremos al perfil de Inspección requerido por el partido en el gobierno, para la ejecución de la política educativa que tiene proyectada.

No obstante todo lo anterior, la fórmula adoptada no conseguirá asentarse en el ordenamiento administrativo y, el PSOE, en 1995, en el proceso de redacción de la LOPEGCE, tendrá que aceptar que se equivocó cuando en 1984 estableció un modelo de Función Inspectora desempeñada de forma no permanente y ajena a un cuerpo de Inspección (ASOCIACIÓN DE INSPECTORES DE EDUCACIÓN DE ANDALUCÍA. (s.f).), e incluirá, en la nueva norma, la creación de un Cuerpo de Inspectores de Educación (LEY ORGÁNICA 9/1995).

Desde 1995 hasta la actualidad, no se han registrado modificaciones en los aspectos que se acaban de tratar. Básicamente, en cada nueva ley de educación, se han 
ido redefiniendo las funciones de las Inspección Educativa, con determinadas modificaciones de ajuste a las demandas del sistema en cada caso.

Por otro lado, cabe mencionar, como paso al siguiente apartado, que desde 1979 se inicia en España el proceso de construcción del Estado de las autonomías en que la educación pasa a ser una competencia plena, pero no exclusiva, dando lugar a que la Inspección Educativa pase a ser un órgano autonómico. Así, queda solamente la Alta Inspección como competencia del Ministerio responsable de Educación, aparte de la Inspección de Educación que se ocupa de los centros educativos en el extranjero, de las ciudades autónomas de Ceuta y Melilla y transitoriamente de las comunidades autónomas de Vía Lenta.

\section{1 - Cambios en el desempeño profesional de la Inspección Educativa en la comunidad autónoma de Andalucía}

Desde aquí, se lleva a cabo una somera presentación de algunos cambios en el desempeño profesional en la Comunidad Autónoma de Andalucía, tras el traspaso de competencias educativas realizado en 1982. Así se puede señalar que:

- La creación de los Equipos de Promoción y Orientación Educativa (EPOE) (DECRETO 238/1983), recoge competencias que ejercían las ponencias de Inspección Educativa.

- La creación de los asesores del profesorado (DECRETO DE LA CONSEJERÍA DE EDUCACIÓN DE 1989) reduce las competencias de asesoramiento pedagógico.

- La creación del servicio de Planificación y Escolarización (DECRETO 270/2005) supone la pérdida de competencias de gestión de cupos de profesorado y, en escolarización, la pérdida de la presidencia de las comisiones de escolarización y la participación en estas. Solo queda la función de supervisión de cumplimiento normativo.

- La creación de la Agencia Andaluza de Evaluación Educativa (AGAEVE) (DECRETO 435/2008), sitúa a la Inspección como colaboradora que participa en la evaluación, con base en los cometidos competenciales establecidos en el eje funcional de evaluación (ORDEN 13/2007) y siguiendo el proceso de intervención indicado en el marco general para la evaluación del sistema educativo andaluz (DECRETO 220/2013). 
- La creación de la aplicación informática Séneca (DECRETO 285/2010), programa de gestión del sistema educativo andaluz, supuso una pérdida de gran dimensión en la función de información a la administración debido a la interconexión que facilita.

Son cambios que se llevan a cabo en Andalucía dentro del periodo de desarrollo autonómico, en que el funcionamiento de la Inspección Educativa, como se ha visto con anterioridad, empezará a ser regulado a partir de 1987, en un proceso que pasa por diferentes fases hasta llegar a la actualidad (SALMERÓN, 2010).

\section{Consideraciones finales}

Pasado el tiempo, observando el recorrido iniciado en los años 6o, se puede contemplar un largo proceso de redefinición del Cuerpo de Inspección que, como se anunciaba al inicio de este documento, ha vivido dos etapas. Una que se extiende de 1960 a 1995; y otra, de 1995 a la actualidad. Treinta y cinco años duró la primera; y veinte, con sensación de mayor continuidad, la segunda.

La primera etapa es de redefinición y se subdivide en dos tramos. Uno, de redefinición orgánica y de funciones, atribuciones y competencias (años 60 y 70) y; otro, de redefinición en la categoría administrativa, al pasar de la concepción de cuerpo de inspección a la de función inspectora (años 80 y primera mitad de los 90). Es una etapa que se puede calificar de búsqueda de la adecuación del servicio de Inspección a las necesidades de modernización del tardofranquismo y las del joven régimen democrático iniciado con la Constitución de 1978. Como se ha podido apreciar por las resistencias ofrecidas desde el propio servicio, desde algunas posiciones ha sido un proceso percibido como de puro descabalamiento del poder detentado y forzada sumisión al poder político, y de menoscabo hacia la alta capacitación técnica del colectivo de Inspección.

La segunda etapa es de consolidación y continua adaptación. La LOPEGCE, en 1995, con la creación del Cuerpo de Inspección Educativa hace efectivos, veinticinco años más tarde, los postulados de la LGE. Da lugar, veinte años después de la muerte de Franco, a la configuración de un órgano perfectamente adaptado a las exigencias de una sociedad democrática y plenamente articulado en la Administración Educativa española, más concretamente, en la estructura de las administraciones educativas autonómicas. Por 
otro lado, actualmente se entiende que el Servicio de Inspección debe estar en continua adaptación de su estructura y funciones, atribuciones y competencias con el objetivo de atender adecuada e inmediatamente a las demandas que el sistema educativo presenta en cada momento. Por ello, es necesaria una configuración abierta y flexible de los procesos que se deben abordar, por lo que es ineludible una excelente y constante cualificación permanente. Pese a lo que se acaba de exponer, cabe señalar que, aunque con escasa incidencia, todavía se expresa la percepción de la existencia de intenciones dirigidas a la minimización y marginación de la presencia de la Inspección Educativa. Si bien, se ha de señalar el incremento de las plantillas en la última década y su presencia indiscutida en el organigrama administrativo de la España actual, lo que se puede percibir como señal de larga continuidad, al haberse convertido en una herramienta imprescindible para favorecer el desarrollo de la calidad educativa.

Por último, en cuanto a las funciones, atribuciones y competencias se ha podido apreciar que las primeras, básicamente se mantienen e incluso aumentan en el transcurso del tiempo, mientras que las atribuciones y competencias dependen, en mayor grado, de los cambios de adaptación y de las demandas originadas por las necesidades del sistema educativo.

\section{Referencias}

BEAS, Miguel. Implantación y desarrollo de la E.G.B. en Granada. Estudio de la creación y funciones de la D.P.E. (1970-1977). Granada: Universidad de Granada, Tesis Doctoral (1993).

BEAS, Miguel. La crisis de la Inspección de Primaria en la década de los setenta. En RUIZ, Julio; BERNAT, Antonio; DOMíNGUEZ, Rosa María; JUAN, Víctor (Eds.). La educación en España a examen (1898-1998), Zaragoza, Ministerio de Educación y Cultura e Institución “Fernando el Católico, vol. I. P. 355-365. (1999).

FENOY, Rafael Historia de la Inspección de Educación: la Inspección de la era de Franco 1936-1970. Disponible en:

http://www.edudactica.es/Docus/Ensayos/Historia3\%20Inspeccion.pdf. (s.f.)

Accedido el 15 de marzo de 2016. 
FENOY, Rafael (s.f.). Historia de la Inspección de Educación: la Inspección Educativa Moderna 1970-2000. Recuperado de:

http://www.edudactica.es/Docus/Ensayos/Historia4\%20Inspeccion.pdf. (s.f.) Accedido el 15 de marzo de 2016.

GÓMEZ, Alberto Luis y ROMERO, Jesús. Reformas educativas y formación Permanente del Profesorado en la última obra de Julia Varela: memoria y olvido. Profesorado: Revista de curriculum y formación del profesorado, 13(1), (p. 231-295), Disponible en:

http://recyt.fecyt.es/index.php/profesorado/article/view/41878/23901. (2009) Accedido el 20 de marzo de 2016.

INFORME FINAL DEL COMITÉ DE COOPERACIÓN INTERNACIONAL PARA LA REFORMA DE LA EDUCACIÓN EN ESPAÑA. Revista de la Hermandad de Inspectores de Enseñanza

Primaria, $n^{\circ} 8$, P. 87-89 (1968).

LÓPEZ del Castillo, $M^{a}$ Teresa. Historia de la inspección de primera enseñanza en España. Madrid: Ministerio de Educación, Cultura y Deporte. (2013).

MAILLO, Adolfo. Historia Crítica de la Inspección Escolar en España. Madrid: Edición Propia. (1989).

MINISTERIO DE EDUCACIÓN Y CIENCIA (MEC). La Educación en España: bases para una política educativa. Madrid: MEC. (1969).

SUREDA, Bernat. Liberalismo y educación del pueblo. Madrid: Biblioteca Nueva. (2006).

\section{Normativa}

Ley 14/1970 de 4 de agosto, General de Educación y Financiamiento de la Reforma Educativa (BOE, de $6-8-70$ ).

Ley 30/1984, de 2 de agosto, de Medidas de Reforma de la Función Pública. Disposición adicional XV (BOE, de 3-08-1984).

Ley Orgánica 9/1995, de 20 de noviembre, de la Participación, de Evaluación y el Gobierno de los Centros Docentes (BOE, de 21-11-95).

Ley Orgánica 10/2002, de 23 de diciembre, de Calidad de la Educación (BOE, de 24-122002).

Ley Orgánica 2/2006, de 3 de mayo, de Educación (BOE, de 4-5-2006).

Ley Orgánica 8/2013, de 9 de diciembre, para la mejora de la calidad educativa (BOE, de 10-12-2013). 
Real Decreto de 20 mayo de 1849, por el que se establece el Reglamento para los Inspectores de Instrucción Primaria del Reino (Gaceta de Madrid n 5315 de 2 de abril de 1849).

Real Decreto de 25 de agosto de 1885, por el que se crea el cuerpo de Inspectores de Primera Enseñanza (Gaceta de Madrid nº 238 de 26-08-1885).

Decreto 2915/1967, de 23 de noviembre, por el que se aprueba el Reglamento del Cuerpo de Inspección Profesional de Enseñanza Primaria del Estado (BOE, de 11-12-1967).

Decreto 2538/1968, de 25 de septiembre, por el que se regulan las Delegaciones Provinciales del Ministerio de Educación y Ciencia y se reorganiza la Inspección General de Servicios del Departamento (BOE, de 18-10-1968).

Decreto 1678/1969, de 24 de julio, sobre creación de los Institutos de Ciencias de la Educación (BOE, de 15-8-1969).

Decreto 664/1973, de 22 de marzo, por el que se regulan las funciones de la Inspección Técnica de Educación (BOE, de 10-4-73). 